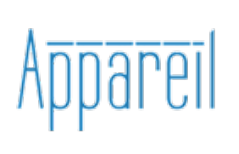

Appareil

12 | 2013

Walter Benjamin. Politiques de l'image

\title{
Éléments pour une anthropologie de l'homme en mouvement
}

\section{Muriel van Vliet}

\section{(2) OpenEdition}

1 Journals

Édition électronique

URL : http://journals.openedition.org/appareil/1983

DOI : 10.4000/appareil. 1983

ISSN : 2101-0714

Éditeur

MSH Paris Nord

Référence électronique

Muriel van Vliet, «Éléments pour une anthropologie de l'homme en mouvement », Appareil [En ligne],

12 | 2013, mis en ligne le 01 décembre 2013, consulté le 30 juillet 2020. URL : http://

journals.openedition.org/appareil/1983; DOI : https://doi.org/10.4000/appareil.1983

Ce document a été généré automatiquement le 30 juillet 2020.

\section{(c) (i) (3)}

Appareil est mis à disposition selon les termes de la Licence Creative Commons Attribution - Pas d'Utilisation Commerciale - Pas de Modification 4.0 International. 


\title{
Éléments pour une anthropologie de l'homme en mouvement
}

\author{
Muriel van Vliet
}

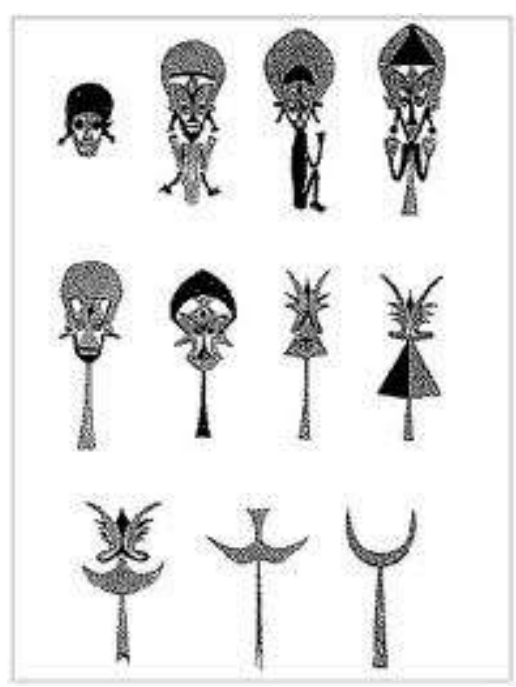

Illustration tirée de Carlo Severi, Le principe de la chimère : une anthropologie de la mémoire?

1 Dans une lettre datée du 15 avril 1924, l'historien de l'art A. Warburg suggère à E. Cassirer, en passe de devenir le philosophe des formes symboliques que l'on connait ${ }^{2}$, que tous deux poursuivent en réalité le même projet : créer " une science générale de la culture comme théorie de l'homme en mouvement ${ }^{3}$ ». Il place ces termes entre guillemets, soit qu'il se cite lui-même, soit qu'il cite des propos récents de Cassirer. Cette lettre répond en effet à l'envoi par le philosophe d'un "symbole consolant" ${ }^{4}$, destiné à la guérison du créateur de la célèbre Bibliothèque hambourgeoise, tragiquement " exilé » de son propre Denkraum pour cause de troubles neurologiques graves. Or ce remède n'est autre que la définition par le penseur Goethe de son projet morphologique, tel qu'il le formule dans Morphologie, formation et transformation des natures organiques. Et cette définition propose de penser la forme comme l'expérience d'un mouvement effectif et efficace créant par oscillation réglée une figure plastique ${ }^{5}$ : 
L'allemand a pour le complexe renvoyant à l'existence d'un être effectif le mot Gestalt. L'allemand produit cette expression par abstraction de ce qui se meut, il suppose qu'un ensemble s'offre ainsi ferme, clos sur lui-même et fixe par nature. Si nous considérons toutefois toutes les figures, en particulier les figures organiques, nous constatons que nous ne rencontrons nulle part quoi que ce soit de consistant, quoi que ce soit qui se trouve en repos, quoi que ce soit de clos sur soi, mais que, bien au contraire, tout vacille dans un mouvement perpétuel. De là l'habitude qu'a notre langue de désigner tant ce qui est produit que ce qui produit par le terme de Bildung. Si nous voulons néanmoins introduire une morphologie, nous devons nous garder du terme de Gestalt, ou, si jamais nous utilisons ce terme, nous devons désigner par là l'idée, le concept ou quelque chose de consistant peut-être, mais seulement dans l'expérience et seulement dans l'instant.

2 Que veut donc désigner Warburg par ce programme commun d'une "science générale de la culture comme théorie de l'homme en mouvement "? Peut-on en dégager les principes, en complétant son approche de l'art avec celle de Cassirer ? Quelles sont les inflexions diverses qu'ils ont pu donner à ce projet et quelle peut en être aujourd'hui l'actualité pour la théorie et pour la politique de l'image ? Cette science recherchée doit-elle être comprise comme le prolongement de la morphologie goethéenne, une lecture des productions culturelles par analogie avec le développement d'organismes vivants en mouvement perpétuel, à la fois "formés" et "formant des séries dynamiques », autrement dit des «formes plastiques », pour mentionner un terme cher également au goethéen que fut aussi Walter Benjamin ${ }^{6}$ ?

Cette «science générale de la culture comme théorie de l'homme en mouvement »se développe selon trois axes intrinsèquement liés qui organiseront notre propos. Elle est à un premier niveau une sémiotique des images, le schéma corporel fonctionnant comme médium symbolique originaire pour créer et recréer les images perçues. À un deuxième niveau, elle enracine continuellement le processus de création du sens des images dans une « symbolisation rituelle ${ }^{7}$ ». Enfin, elle implique à un dernier niveau une critique ou, pour le dire autrement, comme c'est aussi le cas de manière emblématique chez Foucault, elle ouvre sur un "diagnostic critique » implicite qui met au jour le pouvoir de l'image. Ce diagnostic consiste à exhiber rétrospectivement les moments de crise où s'est soudain modifié un ensemble de problèmes spécifiques auxquels tout un groupe culturel s'est confronté et autour duquel il a forgé une unité - cette unité fûtelle par ailleurs traversée par des lignes de force diverses.

En deçà du linguistic turn, qui réduit abusivement le langage à ses seules dimensions représentative et discursive et court ainsi le risque de tomber dans l'écueil de l'« intellectualisme », et au-delà de l'iconic turn, qui, par réaction, déconstruit tellement le sujet créateur et acteur que le domaine de l'éthique s'en trouve dangereusement ébranlé, avec la menace de sombrer cette fois-ci dans l'écueil de «l'esthétisme », l' anthropologie de l'homme en mouvement proposée conjointement par Cassirer et Warburg ouvre la possibilité d'une troisième voie pour le discours sur l'art, replacé dans le cadre d'une théorie de la culture plus globale d'où une nouvelle conception du sujet peut émerger. Sur le modèle des enquêtes ethnologiques centrées davantage sur le rituel que sur le mythe écrit, tous deux perçoivent originairement l'image comme l' expression (Ausdruck) polarisée d'affects opposés, et cela avant même de l'envisager aussi comme la présentation (Darstellung) de valeurs, motifs ou idées que l'artiste comme le spectateur peuvent davantage mettre à distance d'eux que ne l'autorise le niveau expressif pur du rituel magique (Distanzierung, terminologie caractéristique également de la pensée de G.Simmel, un des maîtres de Cassirer qui eut également de 
l'importance pour W. Benjamin). Selon cette anthropologie, art et pensée mythique participent à un processus culturel complexe qui les déborde. La technique, les sciences, l'histoire, le droit, etc. interfèrent constamment pour former des aires relationnelles de projection où s'entre-écrivent différents langages. C'est la mise en évidence des points de basculement d'une aire à une autre qui permet de fournir les éléments d'un diagnostic critique.

Sans placer l'œuvre de Walter Benjamin au centre de la réflexion, l'enjeu implicite de cet essai est de fournir toutefois de la matière pour une évaluation des points de convergence et de divergence potentiels avec celle-ci, selon une comparaison qu'il resterait encore à approfondir au-delà des limites de cet essai. Dans la Petite histoire de la photographie de Benjamin, l'idée de dégager par la photographie " un inconscient optique » pour pouvoir constituer « un atlas pratique » qui joue sur l'agrandissement et l'hétérogénéité des échelles fait implicitement écho à l'Atlas Mnémosyne, réseau mouvant de reproductions photographiques d'images hétérogènes constitué par Warburg dans la seconde moitié des années 1920 à Hambourg pour rénover l'épistémologie de l'histoire de l'art à l'aune d'enquêtes ethnologiques. Les analyses benjaminiennes du rapport dialectique entre mythe et art, entre valeur cultuelle et valeur d'exposition de l'œuvre, mais également ses réflexions, dignes d'un Simmel, sur le rôle du processus d'individuation propre à la création artistique, processus singulièrement complexifié avec l'arrivée de la photographie et du cinéma, peuvent être lues en écho aux travaux de Cassirer sur le rapport dialectique de l'art au mythe, à la religion et à la science. L'élucidation du passage de la fonction cultuelle à la fonction politique de l'art, exposé par Benjamin dans L'œuvre d'art à l'époque de sa reproductibilité technique, peut sans doute bénéficier du contrepoint que fournit le projet warburgien contemporain d'une théorie morphologique de l'image comme mouvement, ou " image-mouvement », la référence à Goethe étant tout aussi centrale chez Benjamin que chez Cassirer et Warburg pour l'élaboration de la notion de forme plastique. Pour définir cette forme, les concepts de transformation, de mouvement et de mise en série dynamique de contenus intuitifs concrets sont en effet solidement articulés par Cassirer, ce qui peut être fructueux pour comprendre en creux l'approche de la politique de l'image développée par Benjamin. Nous proposons donc ici une étude certes « hors champ », si l'on se place du seul point de vue de W. Benjamin et des études cinématographiques, mais qui peut, espérons-le, restituer une partie des références benjaminiennes indirectes ou permettre de travailler des concepts qui lui sont chers pour une potentielle application à des œuvres cinématographiques.

\section{Une sémiotique des images où le corps en mouvement fonctionne comme médium symbolique originaire}

6 Cette partie de notre propos s'appuie sur une série d'articles sur le phénomène d'incorporation dont témoigne la perception des images, selon John Michael Krois, grand spécialiste de Cassirer ${ }^{8}$. Dans ces essais, John Michael Krois insiste sur le fait que pour Cassirer, il n'est pas nécessaire de créer un médium extérieur pour «faire symbole ». Le corps, dans sa dimension expressive, et cela dès le retardement de ses fonctions biologiques nécessaires, est déjà suffisant pour produire de la signification 
culturelle : il est le premier médium symbolique, et ce dès l'expérience motrice de la perception'.

Nous organisons symboliquement notre perception avant même de "penser ». Pour fonder sa phénoménologie, Cassirer remonte à la partition originaire que nous sommes tous capables de faire entre perception de chose et perception d'expression ${ }^{10}$. Cette partition originaire ne peut être fondée ou justifiée par des concepts, elle ne peut qu'être décrite, mais on doit toujours remonter à elle (ainsi qu'à d'autres phénomènes de base, ou Basisphänomene) pour comprendre le processus global de construction du sens. La perception de chose vient historiquement et génétiquement "après " la capacité humaine à percevoir et à reconnaître des expressions, qui apparaît donc comme plus fondamentale. Pour l'homme de la pensée magique qui construit le monde à travers des rituels et n'a pas encore idée de l'abstraction scientifique, la nature entière est perçue comme expressive, qualitative, personnelle. Chaque source prend le visage d'une nymphe, toute la nature est personnifiée par la multitude des « Dieux de l'instant» (Augenblickgötter) qui y résident ${ }^{11}$. Ce n'est que dans un second temps de l'évolution des formes symboliques, avec l'apparition d'une visée proprement théorique (scientifique), que l'homme se met, par opposition avec le mythe, à percevoir la nature comme un ensemble de choses, régies par des lois objectives et selon des unités permanentes (constantes). L'image expressive est très plastique : ce n'est que du point de vue rationnel de la science objective qu'elle se fixe davantage dans un système de représentations (lois), tout en conservant toutefois des traces de son ancrage dans l'image expressive.

8 Si Cassirer part donc bien du langage pour inaugurer sa philosophie des formes symboliques, puisque le premier tome, daté de 1923, porte bien sur le langage et pourrait sembler augurer d'une forme de linguistic turn, le langage est d'emblée abordé non pas seulement et d'abord comme représentatif (comme Darstellung), mais toujours déjà comme expressif (comme Ausdruck) ${ }^{12}$. Un linguistic turn " sémiologique » donc, où le corps signifiant occupe une place première, « avant » la pensée logique qui s'ancre ellemême sur ce premier niveau de construction symbolique pour venir articuler différemment le rapport des parties au tout de l'expérience. Une large place est accordée par Cassirer à l'étude d'un langage affectif, par le biais de l'étude des mimiques, des gestuelles, ce que Warburg désignera comme Gebärdesprache (langage des gestes). Ce protolangage affectif est défini comme construction active d'un premier espace de vie et de pensée (Lebensraum/Denkraum).

9 Cassirer, comme Warburg, est impressionné par le livre pionnier de Darwin consacré à L'expression des émotions chez les hommes et chez les animaux (1872) ${ }^{13}$. Mais s'il admet que l'expression est un moment décisif du langage, il refuse d'en tirer les mêmes conclusions que Darwin, qui naturalise (c'est-à-dire "biologise» strictement) les émotions humaines en les réduisant à n'être que le résidu de réactions mécaniques, réponses à des stimuli naturels. Darwin fait en effet par exemple de la fermeture des poings pour exprimer la colère le reste mécanique d'un geste animal de préhension (chasse) ou de l'exhibition des dents serrées par l'homme en colère le reste mécanique du geste animal de morsure. Contrairement à Darwin, Cassirer pense que les gestes mimiques humains ne sont pas des résultats mécaniques "animaux»: ce sont des traces de la naissance d'une liberté créée par l'espace de vie et de pensée que constitue l'expression humaine. Les mimiques et le langage des gestes sont les résultats premiers d'une fonction symbolique propre à l'homme et à l'homme seul (qu'il qualifie d'animal 
symbolique). Leur étude ne doit pas relever seulement de la biologie, mais aussi de la "sémiotique» (semeiotic), pour reprendre le terme utilisé par C.S. Peirce, destiné à distinguer son approche pragmatique du signe de la linguistique saussurienne. L'approche saussurienne est fondée quant à elle sur l'arbitraire du signe et sur une compréhension binaire du rapport signifiant-signifié ${ }^{14}$. Une conception du langage très différente de la conception saussurienne se dégage au contraire chez Cassirer, tout comme chez Peirce (et W. von Humboldt): le sensible et le sens ne sont pas arbitrairement mis en relation comme " après coup » l'un avec l'autre, mais le sensible et le sens sont intrinsèquement "adaptés » (geeignet), appareillés, originairement l'un à l'autre et n'ont de sens que dans cet appareillage qui est aussi Verkörperung (incorporation) littérale du sens ${ }^{15}$.

10 Or, Cassirer ne se contente pas d'aborder le phénomène d'expression au sein de la forme symbolique que constitue le langage. Pour écrire le deuxième tome de la Philosophie des formes symboliques porté sur la pensée mythique ${ }^{16}$, il travaille en effet dans la célèbre bibliothèque constituée par l'historien de l'art et pionnier de l'anthropologie de l'art que fut A. Warburg, à Hambourg. Il ne connaît pas encore Warburg personnellement, car ce dernier est à ce moment-là malheureusement à Kreuzlingen, dans une maison de repos suisse, soigné par le célèbre psychiatre Ludwig Binswanger, quand Cassirer visite pour la première fois la bibliothèque avec $\mathrm{F}$. Saxl. Ce dernier remplace en effet Warburg à la direction de la bibliothèque de recherche. Les vues de Cassirer et de Warburg vont toutefois se croiser singulièrement, concernant notamment le rapport du langage expressif propre au rituel magique et à l'art. Et c'est d'ailleurs Saxl qui va consolider le lien entre le philosophe et l'historien de l'art.

11 La fonction de sens que constitue l'expression (Ausdruck) est très prégnante quand on considère non pas seulement le langage du corps en général, mais le cas particulier du corps plongé dans l'action que constitue le rituel magique : par exemple le rituel du maïs des Indiens Cora ou la danse du rituel du serpent des Indiens Hopi observée et photographiée par Warburg lors de son voyage d'étude au Nouveau-Mexique chez les Pueblos, à la fin du xix ${ }^{\mathrm{e}}$ siècle $^{17}$.

12 Konrad T. Preuss, ethnologue spécialiste des Indiens Huicholes que Cassirer prend pour guide pour aborder la pensée mythique, centre explicitement son approche non sur le mythe (discursif et potentiellement « écrit »), mais sur le rituel, ce qu'il dénomme avec le terme grec traditionnel de drômenon, ou procession rituelle, acte de déambulation ${ }^{18}$. Le modèle ou prototype pour penser l'activité symbolique humaine dans toutes ses formes n'est pas seulement le corps expressif, mais le corps en mouvement pris dans un rituel collectif, le drômenon. Ce corps expressif en mouvement du rituel crée un espace de vie et de pensée qui permet à un groupe culturel d'élaborer l'espace, le temps et le nombre d'une manière irréductiblement différente, selon les groupes où l'on peut ethnologiquement l'observer.

13 La première « image " à étudier pour aborder le processus entier de création du sens est donc, non pas l'image fixe, comme le sont les premiers totems ou premières idoles, mais le corps expressif en mouvement opérant dans un rituel collectif. Ces cérémonies contribuent autant à créer du lien social qu'à fournir un support pour la mémoire et l'imagination, envisagées non dans leur dimension reproductive, mais déjà créatrice, «libres».

14 L'image est donc pour Cassirer et Warburg ${ }^{19}$ d'emblée et originairement en mouvement. On peut même dire qu'elle n'a, littéralement, même pas à se mettre en mouvement, elle 
l'est par nature et par constitution, dès son origine. Elle correspond à l'incorporation d'une expression qui crée un espace de vie et de pensée permettant d'orienter l'espace, la temporalité d'un groupe culturel déterminé, par contraste avec d'autres.

Or, quel rôle joue l'art (plastique) quand il est envisagé à l'aune de la pensée mythique, dont le rituel serait la caractéristique majeure? Les idoles fonctionnent comme les corps des danseurs du rituel. Elles ont la même " aura ", car elles ont la même valeur cultuelle. Ce sont, pour reprendre la sémiologie ("séméiosis ») de Peirce, des icônes, non des index ou des symboles. De la même façon que le danseur est identifié au dieu le temps que dure la danse magique, de la même façon que la Mort en personne est immédiatement présente quand le mot tabou est prononcé ${ }^{20}$, l'idole est littéralement le dieu, et cela, le temps du rituel (après le rituel, l'idole est comme désacralisée, on s'en désintéresse presque totalement, ce qui troublera, soit dit en passant, les Occidentaux à la découverte du sens de l'art dit " primitif »). L'aura de l'art pris dans un rituel, à quoi tient son pouvoir, est due au phénomène d'identification du signifiant et du signifié ; plus exactement, ils ne sont pas encore distingués et articulés. Signifiant et signifié sont comme concaténés. Cassirer parle ici de concrescence ${ }^{21}$ du signifiant et du signifié, concrescence du sensible et du sens, du visible et de l'invisible (l'anthropologue Carlo Severi usera du terme de "chimère " pour désigner cet appareillage de l'hétérogène dont l'art rituel témoigne, comme nous allons l'approfondir ensuite).

Mais déjà, les idoles de la pensée mythique, par différence avec le pur drômenon où elles prennent leur source, présentent des images qui vont potentiellement pouvoir se dissocier du danseur pris dans le rituel et demeurer à titre de traces de l'acte cérémoniel. Quand elles sont considérées non comme élément du rituel, mais comme art à proprement parler, se produit une nouvelle partition originaire, d'où naissent en même temps la religion et l'art (Cassirer joue sur le double sens du mot allemand Ur-teil qui veut aussi dire «jugement »: il prolonge de manière dynamique l'acte kantien critique de discrimination de différents types de jugement, c'est-à-dire de différents rapports du sujet à l'objet, tout en faisant subir diverses distorsions au kantisme orthodoxe). Avec la religion, l'idole cesse d'être identifiée au dieu : celle-ci n'en est plus que la représentation. Elle est le signifiant, dissociable du signifié qui la « transcende ». On passe de la fonction de sens qu'est l'Ausdruck (caractéristique du rituel magique) à la fonction de sens qu'est la Darstellung, (re)présentation. On pourrait retrouver ici le passage de la valeur cultuelle à la valeur d'exposition que décrit Benjamin, mais elle se joue selon Cassirer dès le passage du mythe à la religion ${ }^{22}$. Du point de vue religieux, on peut même briser l'idole sans porter atteinte au dieu lui-même, qui est au-delà d'elle (iconoclasme protestant). La religion crée une transcendance (un arrière-monde) que le rituel de la pensée mythique ne requérait aucunement pour être complet. Le rituel mythique se produit au plan de la seule immanence. De là d'ailleurs la force et la charge affective "directes»des images que le rituel magique produit: la médiation symbolique humaine est comme "oubliée » par l'homme de la pensée mythique. La religion met quant à elle à distance les images et leur fait perdre leur aura : elle déplace l'aura vers l'idée (le signifié). L'art, quand il est envisagé comme tel et non plus pris dans le rituel magique, articule lui aussi clairement un signifiant et un signifié : l'artiste fait «comme si» par exemple, un danseur était un dieu, mais il n'y a plus d'identification possible avec ce qui est représenté, ce sur quoi joue par exemple Brecht en laissant ses personnages porter des pancartes qui déjouent l'artifice au maximum, pour reprendre un exemple récurrent de W. Benjamin. Mais l'art reste, à la différence de la religion, proche du mythe en cela qu'il reste sur le plan de l'immanence et ne 
prétend pas viser une transcendance au-delà de l'image. L'image est vue comme image et on s'arrête avec l'art à l'image, on fait " arrêt sur image ${ }^{23}$ ». Le jeu entre signifiant et signifié n'est pas aussi strictement codé dans l'art que dans la religion et n'est pas construit au seul bénéfice du signifié (de l'Idée - de Dieu). Le signifié que propose l'artiste est, pour le dire comme Lévi-Strauss, «flottant». L'artiste reste consciemment au seuil du processus de création du sens, sans refermer sa création tragiquement comme le mythe sur la crainte de voir les démons présents et sans ouvrir pour autant sur un signifié transcendant qui ferait du signifiant une coquille vide et sans efficace au plan affectif. $\mathrm{Du}$ point de vue de la conception de l'image, la religion est paradoxalement déjà du côté de la science, elle tend par nature vers l'abstraction: religion et science ne voient en l'image qu'une béquille pour viser une relation idéelle abstraite qui rend l'image relativement arbitraire, quoiqu'indispensable.

Voilà chez Cassirer la dialectique complexe qui se joue entre art, pensée mythique, religion et science ${ }^{24}$. Rituel et art ont tendance à privilégier l'image et sa force affective en restant sur le plan de l'immanence, tandis que religion et science dénigrent l'image sensible pour viser au-delà d'elle et accentuer la relation sensible/sens $d u$ côté $d u$ sens. En reprenant les images "actives » et "efficaces» (« wirksam») du rituel mythique et en ouvrant l'espace de vie et de pensée déjà créé par le corps expressif en mouvement du rituel au lieu de le refermer sur la crainte du divin, l'art est capable de libérer l'homme de ses démons tout en intensifiant l'expression affective sur un plan immanent. John Michael Krois résume cela en disant que « l'art maitrise le mythe pour l'humanitée ${ }^{25}$ ». Warburg dira que le but de l'art est de dé-démoniser les démons qui nous hantent et finissent toujours par revenir. Le rapport de l'art à l'aura est donc complexe et ambivalent. L'art pourrait laisser transparaître un vestige de l'aura mythique (aura qui tenait au phénomène de concrescence de l'expression mythique dans le rituel magique), car il en reste au plan d'immanence, tout en partageant néanmoins avec la religion et la science une même conscience de l'image en tant qu'image et la capacité d'une distance critique (Distanzierung ouvrant sur un possible diagnostic critique implicite ou explicite). L'art serait un rituel mythique avec conscience de la liberté que procure déjà à l'homme la capacité d'expression.

Cela étant dit, quels sont les principaux éléments qui permettent d'envisager la création artistique dans son interaction fructueuse avec le rituel magique?

C'est ici l'historien Warburg et le merveilleux projet de l'Atlas Mnémosyne ${ }^{26}$ qui peuvent venir enrichir concrètement les vues théoriques de Cassirer. Qu'est-ce qui fait non seulement qu'une image puisse se mettre en mouvement, mais qu'il soit de l'essence de l'image d'être toujours déjà en mouvement, puisque le corps expressif en mouvement du rituel magique continue à y jouer le rôle de symbole originaire?

Plusieurs termes-clés s'imposent, que nous proposons de détailler ici sommairement, à savoir : a/ la migration des formules de pathos, l'intensification de la polarité affective (du fait de cette migration), b/l'orientation du schéma corporel qui en résulte et $\mathrm{c} / \mathrm{la}$ structuration de la mémoire, visuelle et conceptuelle, qui en découle. Détaillons ces éléments d'une anthropologie de l'homme en mouvement selon Warburg, en dialogue avec Cassirer.

\section{a/ Wanderung : la migration des formules de pathos}

Ce qui fait qu'une image est toujours déjà en mouvement, c'est qu'elle résulte de la reprise créative d'autres images plus anciennes, relevant donc d'une autre temporalité, et parfois d'un autre espace géographique. La migration produit des tensions 
(Spannungen) entre passé et présent, entre ici et ailleurs, qui font vibrer et osciller les images. Chaque image contient en elle plusieurs points de vue, plusieurs horizons, plusieurs visions du monde qui s'entrechoquent, sont appareillés. L'historien de l'art doit faire resurgir les tensions suscitées par les effets de reprise créative qui sont parfois conscientes aux artistes, mais parfois aussi et le plus souvent en partie inconscientes et ne sont lisibles que rétrospectivement: soit fonctionne en effet une mémoire fondée sur des textes et donc davantage représentative (Darstellung) et plus consciente (l'image illustre un texte) ; soit travaille une mémoire visuelle plus expressive et moins consciente (l'image se modèle analogiquement sur d'autres images). En réalité, ce n'est pas au « ou bien... ou bien... » que nous avons le plus souvent affaire, mais au « et... et... ». En partie, l'artiste cherche explicitement à représenter des mythes (par exemple Goethe, reprenant le mythe de Prométhée et d'Épiméthée dans Pandora); en partie, son œil même est constructivement et activement modelé par les images qu'il a vues lors de son éducation. Son œil peut être considéré comme " constructif », selon les termes de Cassirer, car tout son corps a appris par la "force formatrice » de l'éducation, notamment par l'éducation visuelle, à voir les images activement d'une certaine façon (on retrouve ici des idées d'un autre historien de l'art contemporain à Cassirer et Warburg, j'ai nommé leur collègue et ami Erwin Panofsky). Tradition visuelle et tradition écrite s'entr'expriment, car le corps qui perçoit est le premier prototype de la construction symbolique. Il est lui-même, comme le dira si bien Merleau-Ponty, « entrelacs de vision et de mouvement », c'est-à-dire l'appareillage actif d'une part intellectualisée/intellectualisable de la création du sens et d'une part difficilement intellectualisable sans reste, à savoir le mouvement du corps dans l'espace, créant notamment la profondeur de l'image.

\section{b/ Polarität : polarisation des affects contraires}

Ce qui fait non seulement qu'une image se met en mouvement mais qu'elle est toujours déjà mouvement, tient également au schéma corporel, au montage des cinq sens (synesthésie) et au mouvement du corps percevant. Voir, ce n'est pas voir seulement avec les yeux, fixes comme un appareil photographique, mais voir avec le corps en mouvement, polarisé non seulement selon le haut et le bas, le lointain et le proche, la gauche et la droite, mais aussi selon la joie et la tristesse, l'amour et la haine, la vie et la mort, un ensemble de valeurs "prégnantes ". D'un côté, l'image prête au corps qui la perçoit son schéma organisationnel, de l'autre, le schéma corporel prête son corps à l'image : la relation corps percevant / image perçue est celle d'un chiasme, ce que l'on retrouve sans surprise chez Merleau-Ponty, dont l'une des sources majeures pour La phénoménologie de la perception n'est autre que Cassirer ${ }^{27}$, en particulier lorsqu'il thématise la notion de prégnance symbolique dans le troisième tome de la Philosophie des formes symboliques consacrée à la perception que Merleau-Ponty exploitera ${ }^{28}$. Warburg interprète la polarité réversible des images et du corps qui les perçoit de manière davantage psychologique que Cassirer ou plus exactement, il ne fait pas appel à la même psychologie que Cassirer. Le modèle psychologique de Cassirer, comme celui de Merleau-Ponty, est la psychologie de la forme (Gestaltpsychologie) telle qu'elle est en partie « dé-psychologisée » (et donc «transcendantalisée ») par son cousin neurologue Kurt Goldstein et son collègue Adhémar Gelb ; tandis que le modèle warburgien de la psychologie est la psychologie de la culture de Burckhardt, la psychologie sous-jacente à la philosophie nietzschéenne du soupçon, mais aussi celle de Robert Vischer portant 
sur l'empathie visuelle, et de Binswanger, qui lui transmet des éléments de la psychologie freudienne.

Pour Warburg, non seulement l'image polarise et repolarise dynamiquement les affects, mais elle peut produire des inversions de polarité, phénomène de type freudien que Cassirer ne relève quant à lui pas du tout. Chez Warburg, l'image artistique peut avoir à la fois l'effet dévastateur produit par le rituel, à savoir l'inquiétude, la phobie, la hantise, tout comme elle peut dans le même temps conduire à dé-démoniser les démons. Warburg pense que l'art se situe entre rituel et science. Selon lui, l'image artistique court toujours le risque de l'aspiration dans la tragédie à laquelle peut conduire le rituel, ce dont témoigne par exemple sa manie de la collection des photographies de guerre pendant la première guerre mondiale. Pour Cassirer, l'homme est capable de maitriser pleinement par la création d'images artistiques la polarité affective qui le fait souffrir dans l'image produite par le rituel magique. Poussé ici par les écrits de Goethe et Schiller, Cassirer envisage l'art d'une manière plus apaisée que Warburg ${ }^{29}$. Dès lors que l'historien de l'art met en évidence le principe dynamique de mise en série des motifs qui gouverne silencieusement leur création et régit le groupe de transformation que ces motifs constituent, le pouvoir de l'image est maîtrisé.

L'intérêt de leurs positions est toutefois de ne jamais concevoir le rituel comme source seulement de tragédie, mais déjà comme un espace de vie et de pensée potentiellement libérateur. Pour Cassirer, l'art serait le mythe "moins » la fermeture et la crainte engendrées par l'absence de conscience ou le déni du fait que c'est l'individu créateur qui est la source active de la production de l'image rituelle et qu'il s'agit d'un espace de vie et de pensée que l'homme a créé lui-même, et non pas un Dieu. La perte de l'aura serait bien la clé de l'apparition de l'art authentique, dans la mesure où l'aura marquerait le moment où l'image «faite de main d'homme » oublie qu'elle l'est. On la prétend d'ailleurs souvent " acheiropoietes », c'est-à-dire " non-faite de main d'homme " dans les sociétés dites " primitives ». L'image se met à faire peur à celui-là même qui l'a créée, effaçant malencontreusement le processus d'individuation déjà à l'œuvre au profit d'une pensée clanique où s'abîme la subjectivité ayant pourtant déjà commencé à émerger avec le rituel expressif. Quand l'art reprend la «bonne " partie du rituel, il lui ôte son aura, et en même temps, peut continuer, sur un plan d'immanence, à polariser les affects qui animent le corps pour en faire un microcosme ordonné et ouvrir au processus d'individuation qui garantit à l'homme sa liberté et son autonomie critique. Cassirer bénéficie sur ce point des écrits du sociologue et philosophe Georg Simmel, qui jouera également un rôle pour $\mathrm{W}$. Benjamin.

\section{c/ Orientierung : orientation de l'espace et du temps}

Nous en avons déjà dit quelque chose, mais il convient de rappeler ici que cette orientation n'est pas seulement chez Cassirer et Warburg une question d'orientation affective (constitution d'un espace de vie), mais aussi d'orientation conceptuelle (constitution d'un espace de pensée) ${ }^{30}$. Il peut paraitre étrange de dire que le rituel et l'art servent à penser et non pas seulement à vivre. L'opposition est ici radicale avec un Lévi-Strauss qui dénigre le rituel comme, je cite, un « abâtardissement de la pensée » et relègue son étude à la seule biologie ${ }^{31}$. Au contraire de Lévi-Strauss, Cassirer propose en effet comme Warburg des " catégories de pensée » fondatrices du rituel, élargissant la table kantienne des catégories de manière très hétérodoxe. Rituel et art servent à penser l'espace, le temps et, pour le rituel, pourrait-on ajouter le nombre. Certes, espace, temps et nombre du rituel et de l'art n'ont rien en commun avec espace, temps 
et nombre tels que la science les pense, mais néanmoins, selon Cassirer, il n'est pas possible de comprendre espace, temps et nombre en science sans envisager leur enracinement originaire mythique et artistique ${ }^{32}$.

d/ Mnémosyne : mémoire collective et formation individuelle

L'enjeu de l'examen ample et divers de ces processus de signification n'est autre que la compréhension de la constitution de la mémoire collective et individuelle, affective et scientifique, visuelle et textuelle. Chez Cassirer, cette compréhension s'ancre dans la philosophie goethéenne de la Bildung (formation/culture) et prolonge la réflexion goethéenne sur l'éducation qui est à l'horizon de ces études. Chez Warburg, cela se traduit par un vaste projet de mise en relation de planches qui sont elles-mêmes des réseaux visuels d'images autour de gestes dont on garde mémoire comme la peur, l'enlèvement, la danse... Comment se crée du lien social au-delà d'une simple génération, jusqu'à l'horizon que constitue l'humanité prise comme un tout, dans toute sa diversité créative ? Pourquoi avons-nous pour tâche infinie l'interprétation des traces de l'humain et pourquoi cette tâche interprétative créatrice correspond-elle à l'enjeu politique et éthique de toute science humaine authentique?

\section{La compréhension de l'image à partir du processus de symbolisation rituelle et les enjeux pour la compréhension de la constitution de la mémoire collective}

31 Nous nous appuierons ici sur deux ouvrages de l'anthropologue Carlo Severi : Le Principe de la chimère : une anthropologie de la mémoire et Naven ou le donner à voir (étude écrite en collaboration avec M. Houseman). Nous serons volontairement plus brefs pour pouvoir proposer en conclusion l'analyse d'un exemple concret, une vidéo de l'artiste afghane contemporaine Lida Abdul.

La référence à l'œuvre de Carlo Severi nous permet de revenir à l'idée d'une anthropologie de l'homme en mouvement comme prolongement de la morphologie goethéenne et d'expliciter le caractère « chimérique » de toute création artistique.

Goethe conçoit, comme nous l'avons dit en introduction, la forme comme une forme plastique. La forme est le principe d'une mise en série d'images ou, plus justement peut-être, la mise en série d'images "tout court»: elle est un kaléidoscope. Cet instrument vient d'ailleurs d'être inventé, comme le rappelle Goethe quand il choisit la métaphore du kaléidoscope, pour rendre compte du processus dynamique que l'imagination créatrice de l'artiste doit mettre en œuvre pour, par variation réglée à partir d'un cas saillant ou cas prégnant du réel, faire fructifier cette individualité dense, en en déployant les potentialités latentes ${ }^{33}$.

L'appareillage des planches mobiles d'images de l'Atlas Mnémosyne de l'historien d'art A. Warburg redouble ce processus de création artistique caractérisé par un processus de variation imaginative réglée, c'est-à-dire par la dérivation produite à partir d'un cas " saillant » ou marquant que nous rencontrons et qui nous "arrête ", constituant une sorte de nœud, un point dense, qui suscite en nous une fascination dont nous ne pouvons nous déprendre qu'en l'intégrant activement dans un réseau d'images qu'il va venir faire osciller, qu'il va animer. Il n'est pas étonnant dès lors, soulignons-le au 
passage, que beaucoup d'interprètes aient défini cet Atlas lui-même comme une œuvre d'art à part entière, une installation ou performance, et non comme un chapitre de l'histoire de l'art stricto sensu. Si l'art fonctionne de manière morphologique par mise en séries d'images, l'Atlas Mnémosyne fonctionne comme une "méta-morphologie » qui explicite par son fonctionnement la création artistique elle-même en opérant selon le même processus d'appareillement qu'elle.

Or, c'est cette méthode propre à Warburg que Carlo Severi va explicitement exploiter pour élaborer son propre chantier ethnologique et étudier notamment l'ensemble des comportements que les Iatmüls de Nouvelle-Guinée appellent le rituel du Naven. Or cette étude anthropologique s'appuie sur des modèles inspirés par Warburg et, en amont, se réfère à la morphologie goethéenne qui sous-tend son œuvre tout comme celle de Cassirer. Ce sont ces auteurs qui fournissent entre autres à l'anthropologue des éléments pour l'anthropologie de la mémoire visuelle qu'il élabore en montrant comment l'action complexe du rituel sollicite la fonction symbolique.

Dans Le Principe de la chimère, C. Severi consacre ses premiers chapitres aux études ethnologiques de Warburg portant sur le rituel du serpent. Quand les Indiens Pueblos font fonctionner le symbole en escaliers du serpent-éclair comme trace vivante du rituel du serpent, ils voient à la fois encore en lui s'agiter le véritable serpent qui participe au rituel et ils imaginent déjà sa face "invisible ", c'est-à-dire le concept rationnel de l'éclair qui précède la venue de la pluie. Ce symbole fonctionne comme une " chimère ", un montage ou appareillage entre deux éléments hétérogènes, à savoir entre le choc affectif produit par le serpent dangereux que l'on tient à pleines mains ou à pleine bouche lors du rituel collectif et le concept d'éclair dont on attend le signe apaisant du début de la saison des pluies ${ }^{34}$. Dans sa conférence sur le rituel du serpent, Warburg met alors visuellement en série un ensemble d'images qui forment une variation imaginative réglée complexe dérivée à partir du cas saillant que constitue ce symbole du serpent-éclair. Il appareille ensemble des éléments hétérogènes, sans chercher à réduire par là leur hétérogénéité. Il met au même plan, par exemple, un dessin d'enfant Pueblo rencontré lors de son expédition ${ }^{35}$, la reproduction photographique de la statue gréco-romaine du Laocoon, des photographies du rituel du serpent prises chez les Indiens Pueblos, des photocopies de motifs grecs liés au culte d'Asclépios... Un travail de mémoire se produit par cette mise en série autour d'un cas prégnant qui polarise et condense la série, ou pour employer le langage des mathématiciens, qui intègre les différents éléments rassemblés. Ce travail de mémoire visuel se produit en partie à la manière dont le rêve travaille selon Freud, ou à la manière dont les mythes « se pensent entre eux » selon Lévi-Strauss, qui lui aussi, fautil le rappeler, se réfère à la morphologie goethéenne (et aux phénomènes d'inversion relevés par Freud). Grâce au principe de mise en série (Reihenprinzip), le travail de la mémoire se produit par la manifestation de migrations, de renversements de polarité, de phénomènes d'orientation et de réorientation dont nous avons examiné grossièrement le principe en première partie ${ }^{36}$. Il en résulte des effets de tensions autour de cas prégnants, au cœur des planches d'images, cas saillants qui deviennent de véritables prototypes au sens où ce sont des phénomènes originaires ayant le même statut que la plante originaire recherchée par Goethe : ce prototype ne "supprime " pas ce qu'il met en série en subsumant des cas particuliers sous une règle universelle (selon l'hypotypose schématique kantienne), mais intensifie et fait vibrer toute la série, au point que même une simple photographie insignifiante, tirée d'un journal presque par hasard, semble régie soudain par une forme de "nécessité intérieure" une fois mise 
en série avec d'autres, c'est-à-dire disposée sur la même planche d'images ${ }^{37}$. L'ensemble des éléments hétérogènes mis en série, dont l'individualité n'est aucunement par là réduite, semble régi par une forme interne, pour reprendre le vocabulaire de Goethe ${ }^{38}$. Il y a montage, mais ce montage n'est pas l'effet d'une fantaisie débridée gratuite. Il s'agit d'une «imagination en vue du réel» («Einbildungskraft zur Wirklichkeit») ${ }^{39}$ consciemment mise en œuvre, une imagination qui opère à des classements nécessaires pour organiser le rapport moi-monde, moi-toi.

Si l'on suit le raisonnement poursuivi dans la première partie, il faut mettre en série les images de manière dynamique autour de cas saillants qui deviennent des prototypes, ou nœuds tensifs, points de tension du champ, car la première « image » est celle du corps expressif en mouvement du danseur ou du chaman dans le rituel. Quelque chose de ce mouvement originaire du danseur et du chaman est conservé dans tout phénomène culturel, d'une part parce que nous continuons à voir les images en $\mathrm{y}$ projetant le schéma de notre corps expressif en mouvement et non pas en mobilisant notre seul intellect, qui subsume le sensible sous des concepts, et d'autre part, parce que nous continuons de faire partie d'un groupe social, ne serait-ce que parce que nous sommes individuellement confrontés aux mêmes problèmes que nos contemporains, ces problèmes communs caractéristiques de notre épistémè.

\section{Kulturkritik et diagnostic critique implicite}

En quoi produire ce genre de mise en série pourrait-il impliquer une Kulturkritik ou un diagnostic critique implicite?

On ne trouve pas explicitement de Kulturkritik, ou critique de la culture chez Cassirer, néanmoins, quand on met en relation les trois grands écrits produits pendant la période d'exil américain (1941-1945), on comprend que se dégage une anthropologie qui doit être conçue comme critique de la culture ou comme diagnostic critique «à la Foucaul $\mathrm{t}^{40}$ ». Le montage par Warburg d'éléments hétérogènes sur les planches de l' Atlas Mnémosyne, tout comme les écrits de Cassirer tissant finement les liens entre plusieurs niveaux symboliques pour restaurer la complexité de l'histoire, esquissent des épistémai et marquent plus en profondeur les lignes de fractures entre ces épistémai que l'on aurait pu a priori prédire. Warburg comme Cassirer nous disent que ces lignes de fracture se jouent parfois souterrainement, dans des mutations artistiques, dans des modifications techniques, à partir d'événements apparemment insignifiants, des détails qui font bouger le tableau que constitue l'histoire...

Dans Le Mythe de l'État, Cassirer analyse les mécanismes propres au mythe quand il est utilisé comme moyen de propagande en vue d'établir un État fasciste. Le mythe est consciemment instrumentalisé par des dirigeants violents qui cherchent par-là à désindividualiser les citoyens, à faire du peuple une masse malléable, anonyme, prête à ne plus pouvoir se reconnaitre qu'en un chef fort. Le mythe tel que les Nazis le mettent en œuvre est un moyen puissant pour couper tout le processus de création du sens élaboré par l'ensemble des formes symboliques : il n'y a plus de présentation consciente, car en deçà de toute Darstellung consciente, tout n'est de nouveau qu'expression (Ausdruck) d'affects forts (amour du chef / haine des autres). La valeur d'exposition est réduite à néant, car la valeur cultuelle a repris le dessus. Le chef, ou Führer, se voit doté d'une aura dans les processions militaires : il est le dieu. Il est une incarnation du divin, du sauveur. Mais il n'y a plus comme dans la pensée mythique originaire d'espace de vie 
(sauf au néfaste sens du mot Lebensraum) et de pensée créé par l'expression rituelle : cet espace est au contraire complètement refermé par les Nazis. Le mythe utilisé par les Nazis est paradoxalement utilisé comme les artistes, en faisant «comme si»: les dirigeants ne sont pas les dupes de cette manipulation des foules en vue du pouvoir, grâce à l'expressivité d'images, dont la principale est fournie par la procession militaire. Mais le mythe n'est par là pas libéré et maîtrisé pour l'humanité, les masses ne voient plus le «jeu» (l'écart), le mythe est refermé sur la tragédie qui conduit la masse à élire un tyran qui va la mener à sa perte en la désindividualisant et en la déresponsabilisant. On peut ici parler d'une tragédie de la culture, car c'est bien la masse qui a élu le tyran, maitre des images, qui va transformer les citoyens libres en autant de marionnettes mécaniques. L'imagination de la masse n'est plus une imagination individuelle en vue du réel, mais en vue d'une fantasmagorie dangereuse, menant au gouffre.

41 Toutefois, Cassirer ne veut pas en rester à cette idée d'une tragédie irrémédiable de la culture : la culture est pour lui un drame, non une tragédie ${ }^{41}$. Il serait lâche de s'en remettre au prétendu «destin» des civilisations en pensant que, comme les organismes, elles vivent et meurent, nécessairement et mécaniquement. Le paradigme biologique de la Gestalt ne doit pas conduire à une conception naturaliste de l'homme et le vitalisme est une position contre laquelle Cassirer ne cesse de s'inscrire en faux. C'est l'homme qui crée du sens, ce qui le rend libre. Parce que l'homme reste aux manettes, qu'il est acteur de l'histoire et non la simple marionnette du destin ou des Dieux, il ne tient qu'à lui de redonner foi en l'homme par son action et par la reprise créative notamment de tout l'héritage des Lumières. L'avènement des Lumières est toujours contingent, selon Cassirer, qui déconstruit comme de l'intérieur la philosophie de l'histoire que Kant a élaborée, mais pour reconstruire une pensée de l'individu tout à la fois pris dans le réseau des formes symboliques et acteur de la transformation de ce réseau. La culture est faite de flux et de reflux, son cours n'est pas linéaire et régi par un principe téléologique, aucun absolu logique n'en assure la progression. Cassirer est aux antipodes de Hegel sur ce point. Cette "fragilité des affaires humaines », pour reprendre la belle expression d'Hannah Arendt, n'amoindrit en rien la responsabilité que chaque citoyen doit se donner pour tâche de remplir, bien au contraire. Cassirer défend un optimisme non-naïf permis par la création d'une distance critique, qui est à la fois espace de vie et de pensée et un humanisme que l'on pourrait qualifier d'interminable, pour reprendre le titre d'un article de Patrice Maniglier portant sur Claude Lévi-Strauss ${ }^{42}$. Cassirer aime à citer un bon mot de Goethe, qui pourrait à lui seul illustrer le principe morphologique de l'Atlas Mnémosyne de Warburg: " puisque l'infini nous est à jamais hors d'atteinte ", c'est-à-dire puisque nous devons renoncer à l'absolu et à l'accès à toute forme de transcendance quelle qu'elle soit, "il nous reste à parcourir en tout sens le fini ", à tracer des lignes, à mettre en série les cas individuels par variation imaginative réglée à partir de cas saillants du réel ${ }^{43}$.

Pour conclure, nous voudrions illustrer notre propos avec l'œuvre de l'artiste afghane contemporaine nommée Lida Abdul. Cette artiste, née à Kaboul en 1973, émigrée en Allemagne, puis en Inde et enfin aux États-Unis, travaille actuellement en Afghanistan dans des conditions très difficiles, plusieurs fois menacée de mort à cause de la puissance critique de son œuvre et de son audace. Elle dénonce à travers son œuvre l'islamisme, la guerre, le statut de la femme soumise à l'islam (le port du voile, l'état de minorité où on la réduit) ${ }^{44}$. 
Elle a réalisé en 2005 une œuvre nommée White house. Cette œuvre est une vidéo d'environ six minutes qui montre d'abord une image qui semble fixe: celle d'un bâtiment en ruines. Il s'agit d'un bâtiment situé non loin du Palais royal, un ancien bâtiment d'allure gréco-romaine. Le mythe est en ruines. Les dieux ont déserté les lieux. Le rituel consistant à repeindre les murs en blanc comme symbole de pureté, de pouvoir, d'absolu, ne peut plus avoir de sens. Et pourtant, l'image se met en mouvement d'abord du fait de petits éclairs qui déchirent le ciel gris sombre au loin, à l'arrière-plan, comme souvenir de l'aura des dieux qui ont déserté le paysage. Il n'y a plus rien d'absolu, nous sommes sur le plan d'immanence, l'homme est face à lui-même. Tout à coup, nous assistons à une rupture de plan et à une brusque inversion de polarité : le bâtiment est devenu blanc, même le sol autour du bâtiment est blanc. Il n'a plus valeur cultuelle, l'aura a disparu, mais il prend clairement valeur d'exposition et valeur politique. Peint consciencieusement depuis trois jours par l'artiste, le tas de ruines est devenu une œuvre d'art, mais il le devient surtout parce que l'œuvre de Lida Abdul est une vidéo. Un dromenôn est rejoué par l'artiste pour libérer le sens du rituel consistant à repeindre un bâtiment politique ou religieux en blanc. C'est une femme qui peint, non un homme: Lida Abdul elle-même. Le spectateur assiste à une première inversion de polarité affective. Le bâtiment est transformé par sa présence. Elle peint avec grand sérieux et grande application, avec le sérieux du rituel. Mais nous ne sommes pas seulement dans un rituel. L'artiste procède par variation imaginative réglée: son imagination travaille non de façon débridée, mais avec une forme de nécessité intérieure, avec systématicité. Elle fait collection des cailloux rencontrés en les intégrant à sa mise en série. Arrive un homme : il est comme elle habillé de noir, ce qui rappelle les habits du deuil, mais ici surtout le sérieux du diagnostic critique implicitement engagé. Le rituel de peindre en blanc des colonnes est rejoué de manière dérisoire, mais ce n'est pas un jeu : c'est un engagement politique très clair. Lida Abdul peint le dos de l'homme en blanc, comme si l'homme était lui aussi en ruines et devait être transformé, cette purification par le blanc n'ayant plus le rôle d'ouvrir vers l'absolu du divin (vers la transcendance de la toute puissance politique), mais seulement d'ouvrir à la conscience critique, au plan d'immanence. Un rapport d'homme à homme sans violence est encore possible parmi les décombres, un geste de douceur venu d'une femme qui doit normalement porter le voile et laisse ici librement flotter ses cheveux au vent, ce qui n'est pas sans écho avec les analyses de Warburg sur le pathos augmenté par le mouvement de petits détails (le mouvement des cheveux, des vêtements, etc., tel qu'il l'analyse en particulier pour la Naissance de Vénus " antiquisante» de Botticelli ${ }^{45}$ ). C'est la femme qui agit, qui est l'acteur principal, l'homme est passif et sa passivité fait ici sa noblesse, non sa médiocrité. Nouvelle inversion de polarité, donc. Il accepte de se laisser peindre au milieu des ruines par une femme pour laisser espérer de nouveaux rapports homme/femme, mais aussi, en général de nouveaux rapports humains, car ce n'est pas tant l'amour entre un homme et une femme qui est ici suggéré, qu'une fraternité humaine plus profonde. Il y a perte d'aura, perte du lien homme/dieux, mais quelque chose de la beauté de l'aura survit dans la beauté de la relation homme/homme. Une foi en l'homme s'affirme courageusement, avec une grande prise de risque, une forme d'humanisme. Il ne s'agit bien sûr pas de l'humanisme conservatiste, frileux, rétrograde, élitiste, fermé vers le passé, mais de l'humanisme ouvert vers l'avenir de créations futures, vers toutes ces «aurores nouvelles qui n'ont pas encore luit » qu'invoque Nietzsche: un humanisme tourné vers l'avenir plutôt que vers le passé. 
L'anthropologie de l'homme en mouvement veut aussi dire ceci chez Cassirer et Warburg : il reste encore à inventer, tout n'est pas encore inventé, de nouvelles formes peuvent, doivent émerger. Un grand optimisme et une grande modestie se dégagent des pensées de Warburg et de Cassirer. Ce doit être notre tâche, ne pas abandonner notre grande créativité formelle. En tant qu'individus, notre tâche consiste à être activement tournés vers cette créativité de formes nouvelles, être des «seconds Prométhée ». Comme le dit Lévi-Strauss, ce n'est pas parce que la conscience n'est pas tout que nous devons renoncer à prendre conscience. Au contraire, c'est notre tâche, mais une tâche par définition interminable, car de nouvelles formes doivent encore émerger... Et ici, Warburg et Cassirer rejoignent les perspectives de W. Benjamin, selon des modalités qu'il faudrait, sur la base des éléments que nous avons essayé de rassembler dans cette présentation, bien sûr davantage préciser.

\section{BIBLIOGRAPHIE}

Cassirer Ernst, Philosophie des formes symboliques, t. I, Le langage, Ole Hansen-Love et Jean Lacoste (trad.), Paris, Minuit, 1972.

Cassirer Ernst, Philosophie des formes symboliques, t. II, La pensée mythique, Jean Lacoste (trad.), Paris, Minuit, 1972.

Cassirer Ernst, La philosophie des formes symboliques, t. III, La phénoménologie de la connaissance, Claude Fronty (trad.), Paris, Minuit, 1972.

Cassirer Ernst, Essai sur l'homme, Norbert Massa (trad.), Paris, Minuit, 1975.

Cassirer Ernst, Le Mythe de l'État, Bertrand Vergely (trad.), Paris, Gallimard, 1993.

Cassirer Ernst, Liberté et forme : l'Idée de la culture allemande, CEuvres XLV, Jean Carro, Martha Willmann-Carro et Joël Gaubert (trad.), Paris, Éditions du Cerf, 2001.

Cassirer Ernst, Logique des sciences de la culture, Jean Carro et Joël Gaubert (trad.), Paris, Éditions du Cerf, 2007.

Cassirer Ernst, Nachgelassene Manuskripte und Texte, Band 18, Ausgewählter wissenschaftlicher Briefwechsel, John Michael Krois (éd.), avec la participation de Marion Lauschke, Claus Rosenkranz et Marcel Simon-Gadhof, Hambourg, Meiner Verlag, 2009.

Darwin Charles, The Expressions of the Emotions in Man and Animals, London, 1872. Déotte JeanLouis, Walter Benjamin et la forme plastique : architecture, technique, lieux, Paris, L'Harmattan, 2012.

Didi-Huberman Georges, L'image survivante, Histoire de l'art et temps des fantômes selon Aby Warburg, Paris, Minuit, 2002.

Hagelstein Maud, «L'art, entre mythe et science chez Cassirer et Warburg », in Cassirer et l'art comme forme symbolique, Rennes, Presses universitaires de Rennes, 2010.

Houseman Michael, Severi Carlo, Naven ou le donner à voir, Paris, CNRS/Éd. de la Maison des sciences de l'Homme, 2009. 
Krois John Michael, «Introduction », in Ernst Cassirer, Écrits sur l'art, Christian Berner, Fabien Capeillères, Jean Carro et Joël Gaubert (trad.), Paris, Éditions du Cerf, 1995.

Krois John Michael, Bildkörper und Körperschema [Corps de l'image et schéma corporel], Berlin, Akademie Verlag (Actus et Imago), 2011.

La forme selon Ernst Cassirer - De la morphologie au structuralisme, Muriel van Vliet (dir.), Rennes, Presses universitaires de Rennes, 2013.

Lévi-Strauss Claude, Les Mythologiques. L'homme nu, Paris, Plon, 2009.

Lida Abdul, Torino, hopefulmonster editore, 2007.

Maniglier Patrice, «L'humanisme interminable de Claude Lévi-Strauss », Les Temps Modernes, $\mathrm{n}^{\circ}$ 609, juin-août 2000, p. 216-241.

Severi Carlo, Le Principe de la chimère : une anthropologie de la mémoire, Paris, Éd. Rue d'Ulm/musée du quai Branly(Aesthetica), 2007.

Warburg Aby, " "La naissance de Vénus" et "Le printemps” de Sandro Botticelli », in Essais Florentins, Sibylle Müller (trad.), Paris, Klincksieck, 1990.

Warburg Aby, Gesammelte Schriften, II, 1, Der Bilderatlas Mnemosyne, Martin Warnke, Claudia Brink (Éds.), Berlin, Akademie, 2000.

Warburg Aby, Miroirs de faille - À Rome avec G. Bruno et É. Manet, 1928-29, Maurizio Ghelardi (Éd.), Sacha Zilberfarb (trad.), Paris, Presses du Réel, 2011.

Warburg Aby, Le Rituel du serpent, Paris, Macula, 2011.

Warburg Aby, L'Atlas Mnémosyne, avec un essai de Roland Recht, Sacha Zilberfarb (trad.), Paris, Presses du Réel, 2013.

\section{NOTES}

1. L'illustration est tirée de Carlo Severi, Le Principe de la chimère: une anthropologie de la mémoire, Paris, Éd. Rue d'Ulm/musée du quai Branly (Aesthetica), 2007, p. 54, un ouvrage auquel il sera fait référence ultérieurement. Les arguments de cet article ont été présentés le 11 décembre 2012 lors d'une séance au séminaire de Jean-Louis Déotte et d'Alain Naze à la MSH Paris Nord. Nous les remercions ici vivement de nous avoir ainsi permis de mettre en forme ces idées.

2. Les trois tomes de la Philosophie des formes symboliques consacrés au langage, au mythe, à la perception sensible et à la science datent respectivement de 1923, 1925, 1929.

3. Voir la riche correspondance d'Ernst Cassirer, Nachgelassene Manuskripte und Texte, Band 18, Ausgewählter wissenschaftlicher Briefwechsel, édité par John Michael Krois, avec la participation de Marion Lauschke, Claus Rosenkranz et Marcel Simon-Gadhof, Hambourg, Meiner Verlag, 2009, abrégé ECN 18. La citation provient de : Aby Warburg à Ernst Cassirer, lettre du 15 avril 1924, ECN 18, p. 67. Nous traduisons in extenso : « [...] l'ellipse <sous-entendu le projet képlérien> est le point de départ ou encore la ligne de partage qui marque un changement de climat [Wetter], si nous voulons tous deux inaugurer "une science générale de la culture comme théorie de l'homme en mouvement" ". Il veut dire que leur projet s'enracine dans l'humanisme de la Renaissance qui a permis un nouveau rapport de l'individu au cosmos et une nouvelle compréhension de la liberté humaine.

4. Ernst Cassirer à Aby Warburg, lettre du 15 avril 1924, ECN 18, p. 65.

5. Ernst Cassirer à Aby Warburg, lettre du 12 avril 1924, ECN 18, p. 65. 
6. Tel qu'il est mis à l'honneur dans le dernier livre de Jean-Louis Déotte, Walter Benjamin et la forme plastique: architecture, technique, lieux, Paris, L'Harmattan, 2012, p. 87 sur les Gestaltungsformen.

7. Michael Houseman, Carlo Severi, Naven ou le donner à voir, Paris, CNRS/Éd. de la Maison des sciences de l'Homme, 2009, p. 219 et suiv.

8. John Michael Krois dirigeait à Berlin, avec l'historien de l'art Horst Bredekamp, un centre d'étude sur l'image nommé « Bildakt und Verkörperung " ("L'image comme acte - ou l'acte-image et le phénomène d'incorporation »). Il y proposait notamment un séminaire sur la philosophie du film, mettant librement les concepts cassireriens, warburgiens et panofskiens en application. John Michael Krois s'est malheureusement éteint brutalement en 2010. Le premier essai sur lequel nous nous appuyons est intitulé «Cassirer's 'prototype and model' of symbolism » [ "Prototype et modèle du symbolisme chez Cassirer »]; le deuxième « Die Universalität der Pathosformeln - Der Leib als Symbolmedium» [L'universalité des formules de pathos - Le corps comme médium symbolique »] paru d'abord dans l'ouvrage collectif Quel corps ? Eine Frage der Repräsentation, Hans Belting, Dietmar Kämper et Martin Schulz (éd.), Munich, Wilhelm Fink Verlag, 2002 ; le troisième est intitulé "More than a Linguistic Turn in Philosophy. The Semiotic Programs of Peirce and Cassirer " [«Plus qu'un tournant langagier en philosophie. Le programme sémiotique de Peirce et de Cassirer »]. Ils figurent tous trois dans la publication posthume intitulée Bildkörper und Körperschema [Corps de l'image et schéma corporel], Berlin, Akademie Verlag (Actus et Imago), 2011.

9. John Michael Krois, Bildkörper und Körperschema, p. 52.

10. Ernst Cassirer, Logique des sciences de la culture, traduit par Jean Carro avec la collaboration de Joël Gaubert, Paris, Éd. du Cerf, 2007, abrégé LSC, p. 119: «Il n'existe aucune perception qui ne s'adresse à un "objet" précis et ne soit orienté vers lui. Mais cette indispensable référence à l'objet se présente pour nous dans une double orientation que nous pouvons brièvement et schématiquement décrire comme l'orientation vers le "ça" et l'orientation vers le "toi". [...] Il est incontestable que l'homme vit la réalité sous ce double aspect ".

11. Ernst Cassirer, LSC, p. 120 : «Le primat <de la perception d'expression> sur la perception des choses est la caractéristique proprement dite de la vision mythique du monde. Il n'y a pas encore de "monde des faits" rigoureusement défini et distinct, car il lui manque encore ces unités constantes que toute connaissance théorique vise à obtenir en premier lieu. Chaque figure peut se changer en une autre et tout sortir de tout. La forme des choses menace à chaque instant de devenir floue, car elle ne s'édifie pas sur des propriétés permanentes. [...] Pour <la pensée mythique> le monde peut à chaque instant prendre un autre visage, car c'est l'affect qui définit ce visage. Dans l'amour et la haine, l'espoir et la crainte, la joie ou l'effroi, les traits de la réalité se modifient. Chacune de ces excitations peut donner naissance à une nouvelle figure mythique, un "dieu du moment" ". Cassirer renvoie ici en notes à son essai Sprache und Mythos. Nous soulignons.

12. Ernst Cassirer, Philosophie des formes symboliques, t. I, Le langage, Ole Hansen-Love et Jean Lacoste (trad.), Paris, Minuit, 1972.

13. Cassirer utilise l'édition anglaise suivante: Charles Darwin, The Expressions of the Emotions in Man and Animals, London, 1872. Voir le commentaire circonstancié qu'en donne G. DidiHuberman, L'image survivante, Histoire de l'art et temps des fantômes selon Aby Warburg, Paris, Minuit, 2002, voir notamment les illustrations qu'il choisit p.228-229, p. 232-233 et p. 239. Notre compréhension du rapport de Cassirer à Warburg se distingue néanmoins de celle de G. DidiHuberman. Voir Muriel van Vliet, La forme selon Ernst Cassirer - De la morphologie au structuralisme, Rennes, Presses universitaires de Rennes, 2013.

14. John Michael Krois, Bildkörper und Körperschema, p. 94 : «The point of Pragmatism was not that it took its departure from the utilitarian considerations of 'usefulness'; practicality was a secondary matter, for practicality depended upon the way things behaved. The point was rather that philosophy needed to begin with embodiement - the embodiement of thought in signs, of belief in habits of action, and the "mind" in the body ». P. 95: "With the prominent exception (in the English-speaking world) of Nelson 
Goodman, signs and symbols other than language and the semiotic character of perception itself have been largely ignored by philosophers. In particular, most philosophers limit the investigation of meaning to language. Charles Peirce and Ernst Cassirer did not ». Note 5, p. $95:$ : Peirce preferred to spell the name of the theory of signs and symbols as "semeiotic", which offers a way to distinguish his theory; Saussure named his theory "sémiologie" ".

15. Le pragmatisme de Peirce trouve un accord avec Cassirer sur ce point, voir ces citations de Cassirer qui permettent d'appuyer l'hypothèse d'un rapport de Cassirer avec un certain pragmatisme : «Un objet se résume à être ce en tant que quoi seulement il se donne à nous : une somme de modalités d'actions effectives et possibles »; "Ce ne sont pas de pures observations, mais plutôt les actions qui sont au centre où, pour les hommes, l'organisation intellectuelle de la réalité s'origine », toutes mentionnées par John Michael Krois, idem, p. 97.

16. Ernst Cassirer, Philosophie des formes symboliques, t. II, La pensée mythique, Jean Lacoste (trad.), Paris, Minuit, 1972, abrégé PFS II.

17. Aby Warburg, Le Rituel du serpent, Paris, Macula, 2011.

18. Ernst Cassirer, PFS II, p. 61 : «Ce n'est pas un simple spectacle, une simple pièce, qu'exécute le danseur qui participe à un drame mythique : le danseur est le dieu, il devient le dieu. Tous les rites de la végétation en particulier, dans lesquels on célèbre la mort et la résurrection du dieu, expriment ce sentiment essentiel de l'identité, cette identification réelle. Ce qui a lieu dans ces rites, comme dans la plupart des rites mystérieux, n'est pas une simple mise en scène qui imiterait un événement, mais l'événement lui-même, son accomplissement immédiat : c'est un drômenon, un événement réel et effectif, parce que parfaitement efficace ». Cassirer cite Preuss, se référant lui-même à Usener: "le but recherché n'est jamais de présenter des scènes et des pensées. Cela ne peut arriver que lorsque les danses sont devenues profanes ou qu'elles ont atteint un haut degré d'évolution » (Preuss, Religionen der Naturvölker, 1904).

19. Ainsi que nous l'a fait remarquer récemment Maud Hagelstein, si Warburg travaille à partir d'images fixes, c'est toujours pour reconstituer le système de tensions, le jeu de ressemblances et de différences qui se joue entre les œuvres étudiées, de sorte que c'est un mouvement virtuel qui est mentalement et affectivement à restituer. Selon Warburg, les œuvres appellent une polarisation.

20. Ernst Cassirer, PFS II, p. 62 : Dans la pensée mythique, «le mot et le nom eux non plus, ne désignent pas et ne signifient pas - ils sont et ils agissent». PFS II, p. 278: «Bien avant par conséquent que le signe écrit soit conçu comme l'expression d'un objet, il est entouré de crainte, dans la mesure où l'on voit en lui l'ensemble substantiel de tous les effets que l'objet peut produire, c'est-à-dire une sorte de double démoniaque de l'objet », Cassirer se réfère ici à Th. W. Danzel.

21. Ernst Cassirer, PFS II, p. 88 : «La pensée mythique se révèle en cela "concrète" au sens strict : ce qu'elle saisit subit alors une concrétion spécifique, qui le fait s'agglomérer en se développant (con-crescere). La connaissance scientifique s'efforce de conclure à la cohésion des éléments distincts, alors que l'intuition mythique laisse en dernière analyse coïncider ce qu'elle lie. L'unité de la liaison, comme unité synthétique, et donc comme unité du divers, est remplacée par la singularité d'une chose, la coïncidence, et cela se comprend quand on songe qu'il n'y a au fond pour la relation dans la vision mythique qu'une seule dimension, qu'un seul plan d'être ». Le mythe «ne connaît que l'existence et l'action immédiates. Les relations qu'il pose par conséquent ne sont pas des liaisons intellectuelles grâce auxquelles on sépare et on réunit en même temps les éléments qui entrent en elles : c'est au contraire une sorte de ciment qui peut toujours agglomérer de quelque manière ce qu'il y a de plus hétérogène ". Nous soulignons.

22. Tandis que Cassirer se place du point de vue d'une anthropologie générale, Benjamin expose les conditions matérielles qui sont à la source d'une nouvelle politique de l'image caractéristique de la modernité, ce qui rend certes difficile d'établir une réelle comparaison entre ces deux penseurs, préoccupés néanmoins par certains thèmes communs. 
23. Ernst Cassirer, PFS II, p. 304 : «Car ce qui définit précisément la direction fondamentale de l'élément esthétique est le fait qu'il reconnaît l'image en tant que telle et que, pour remplir sa fonction, celle-ci n'a pas besoin de rien abandonner d'elle-même et de sa valeur propre. Le mythe voit toujours dans l'image un fragment de la réalité substantielle, une partie du monde des choses lui-même, qu'il dote de forces égales ou supérieures à celles de ce monde. La conception religieuse s'efforce de donner une forme spirituellement plus pure à cette vision magique. Et, pourtant, elle se voit elle aussi toujours conduite à un point où le problème de son sens et de sa vérité se renverse brusquement et devient le problème de la réalité de son objet, un point où, dur et abrupt, se dresse devant elle le problème de l'“existence". Seule la conscience esthétique abandonne véritablement le problème. Pour que les images projetées par cette conduite de la conscience acquièrent une signification purement immanente, il faut que celle-ci s'abandonne dès l'origine à la pure "contemplation" <logique des couleurs et des formes pures>, et qu'elle élabore la forme de la vision théorique en l'opposant à toutes les autres formes de l'activité <la contemplation étant elle aussi envisagée comme une forme d'activité> [...] La régression vers cette légalité donne naissance, simultanément, à une liberté nouvelle de la conscience : l'image n'est plus une chose autonome qui agit par réaction sur l'esprit : elle est devenue pour lui la pure expression de sa force créatrice ".

24. Voir ici l'essai de Maud Hagelstein sur "L'art, entre mythe et science chez Cassirer et Warburg ", dans Cassirer et l'art comme forme symbolique, recueil collectif que nous avons eu la chance de pouvoir diriger pour les Presses universitaires de Rennes en 2010. Maud Hagelstein est l'une des principales spécialistes de Warburg, et travaille également sur Benjamin et Kracauer dans son séminaire d'esthétique, à Liège.

25. John Michael Krois, « Introduction », in Ernst Cassirer, Écrits sur l'art, Christian Berner, Fabien Capeillères, Jean Carro et Joël Gaubert (trad.), Paris, Éditions du Cerf, 1995, p. 8.

26. Voir Aby Warburg, Gesammelte Schriften, II, 1, Der Bilderatlas Mnemosyne, Martin Warnke, Claudia Brink (Éds.), Berlin, Akademie, 2000. Une traduction française de l'introduction est d'abord parue dans Aby Warburg, Miroirs de faille - À Rome avec G. Bruno et É. Manet, 1928-29, Maurizio Ghelardi (Éd.), Sacha Zilberfarb (trad.), Paris, Presses du Réel, 2011, p. 139-152, p. 140 concernant « le processus de dé-démonisation du patrimoine héréditaire des impressions phobiques ", désormais, le public francophone dispose aussi de Aby Warburg, L'Atlas Mnémosyne, avec un essai de Roland Recht, textes traduits par Sacha Zilberfarb, Paris, Presses du Réel, 2013.

27. Voir sur ce point notre article dans Cassirer et l'art comme forme symbolique, Rennes, Presses universitaires de Rennes, 2010.

28. Nous citons Carlo Severi, Le principe de la chimère, p. 37-38: «[...] Percevoir est [...] toujours projeter une image de soi. [...] l'esprit projette toujours dans l'imagination une représentation symbolique du corps. [...] Toute perception est pour $<$ Warburg $>$ le produit d'une mise en relation inconsciente entre l'image extérieure -ou plutôt sa forme - et cette activité de projection incessante qui fait partie de la perception visuelle $»$.

29. En tout cas, c'est le cas dans la Philosophie des formes symboliques. On peut relever toutefois d'autres inflexions encore dans le Mythe de l'État, écrit pendant l'exil américain, pour démonter l'instrumentalisation du «mythe " par les Nazis.

30. Comme nous l'a fait remarquer Maud Hagelstein, Warburg considère l'orientation comme une prise de position au sens quasi-politique où il s'agit de se positionner par rapport à des modes d'explication du monde.

31. Claude Lévi-Strauss, Les Mythologiques. L’homme nu, Paris, Plon, 2009, p.603. Cité par John Michael Krois, Bildkörper und Körperschema, p. 87.

32. Voir sur ce point l'essai de 1931 sur «Espace mythique, espace artistique, espace scientifique ", Écrits sur l'art, p. 101 et suiv. Sur cet essai, voir l'article de Jean Seidengart, «Espace esthétique et espace cognitif dans la philosophie de la culture d'Ernst Cassirer », dans l'ouvrage collectif Cassirer et l'art comme forme symbolique, p. 145 et suiv. 
33. Cassirer cite Goethe dans Liberté et forme : l'Idée de la culture allemande, Euvres XLV, Jean Carro, Martha Willmann-Carro et Joël Gaubert (trad.), Paris, Éditions du Cerf, 2001, p. 233 : «J'avais ce don, quand je fermais les yeux, pour me représenter une fleur au centre de mon organe de la vue, que celle-ci ne se figeait pas un seul instant sous sa forme première mais elle s'ouvrait et dans son centre s'épanouissaient de nouvelles fleurs aux pétales colorés et feuilles vertes; ce n'étaient pas des fleurs naturelles mais des fleurs imaginaires, bien que régulières comme les rosaces d'un sculpteur. Il était impossible de fixer cette création jaillissante, elle durait en revanche aussi longtemps que je le désirais, sans gagner ni perdre de sa force. Je pouvais de même, quand je me représentais un vitrail, faire naître son décor polychrome qui ensuite se transformait sans cesse depuis son centre jusqu'à la périphérie, à l'image du kaléidoscope récemment inventé [...] C'est ici que peut commencer directement l'examen plus conséquent de tous les beaux-arts; on voit clairement ce que veut dire l'idée que les poètes et tous les vrais artistes doivent être nés tels. Leur force créatrice interne doit en effet sortir d'elle-même, sans préméditation et volonté, ces images, ces idoles conservées dans les sens, dans le souvenir, dans la force d'imagination: elles doivent s'épanouir, grandir, s'épandre et se condenser pour devenir, d'ombres fugitives qu'elles sont, des essences véritablement objectives » (Das Sehen in subjektiver Hinsicht, Naturwiss. Schr., XI, p. 282).

34. Carlo Severi, Le Principe de la chimère, p. 41 : «Comme Vischer l'avait pressenti, Warburg découvrait alors que la représentation mentale liée à une trace matérielle inscrite sur un support (un dessin) peut excéder ce que l'image donne à voir ». Il s'agit pour Warburg de "retrouver le "squelette héraldique de la forme". Un oiseau, par exemple, est décomposé dans ses parties essentielles, de manière à apparaître comme une abstraction héraldique. Il se change en hiéroglyphe, qui n'est plus seulement fait pour être contemplé, mais pour être déchiffré. Nous nous trouvons devant "un compromis entre image et signe, entre image-reflet réaliste et écriture" " (Aby Warburg, Le Rituel du serpent). Quand Warburg interprète les poteries hopi, "l'image-signe de l'oiseau hopi était le résultat d'une composition associant des éléments hétérogènes, et non seulement des "parties du corps" de l'oiseau. Pour représenter les êtres surnaturels dans l'art de la poterie, les Hopi utilisent un schéma iconographique qui associe, comme la Chimère des Grecs, l'image d'éléments différents dans un seul corps. [...] Warburg découvrait ainsi que ces enfants qui représentaient la foudre à partir de l'image d'un serpent dans le ciel reprenaient exactement le même procédé d'articulation, en un seul corps, d'éléments désignant des êtres différents. Le Serpent-foudre comme la représentation hopi de l'oiseau en forme de hiéroglyphe, ce "compromis entre signe et image", étaient en réalité des chimères ".

35. Il demande à cet enfant de représenter un éclair et ce dernier le symbolise curieusement comme le serpent-éclair esquissé sur le sable de la kiwa rituelle, là où des serpents jetés avec violence rendront l'image magique efficace car vivante.

36. Carlo Severi détaille le travail morphologique de Pitt-Rivers, avec, en 1874, un ouvrage intitulé Principles of classification. Ce dernier procède à rebours du biologiste: il cherche à reconstruire le passé à partir des traces que l'organisme évolué peut en révéler. Il faut " prophétiser le passé ». Pitt-Rivers s'inspire de Goethe pour sa " psychologie des arts matériels » (p. 52). Selon lui, «les opérations mentales se perpétuent dans le temps par l'évolution des formes ", leur étude permettant une zoologie ou botanique des idées. D’où ces planches : série morphologique de l'évolution du boomerang, série morphologique de l'évolution de la pagaie en Nouvelle-Irlande » que C. Severi reproduit p. 53-54 de son livre. Pour sa part, C. Severi critique la conception de certains continuateurs de Pitt-Rivers, tel Henry Balfour. Selon lui, il faut une morphologie qui ne soit pas dénuée d'ambition psychologique: il faut se référer à la pensée visuelle, quitte à rester ambigu sur l'articulation des deux plans, psychologiques et formels.

37. Carlo Severi, Le Principe de la chimère, p. 63 : «Le succès d'une image, qui signifie à la fois son intensité et sa capacité à devenir le prototype d'une série menant à des cryptoglyphes s'explique » par un double processus de simplification et de projection. P. 61 : on peut parler d'un 
acte de regard « qui va bien au-delà de la simple imitation de la forme. On passe de l'idée d'une imitation d'un objet naturel à celle d'une interprétation [...]. L'analyse d'un objet permet donc de retrouver la trace d'un exercice actif de la perception, d'un acte de l'imagination opérant selon le principe que Vischer avait appelé empathie visuelle ». Il y a comme un dialogue " entre le regard et la forme naturelle. Comme dans la chimère hopi, qui associe les traits d'un serpent à ceux de la foudre, l'image en tant que trace matérielle fait émerger un travail de l'esprit, une série d'opérations mentales qui se trouvent associées à l'image ».

38. Kandinsky réutilisera dans Du spirituel dans l'art et dans la peinture en particulier l'expression de forme interne ou de nécessité intérieure pour parler de l'effet des couleurs, mises en série ou plus exactement disposées en cercle. Le cercle chromatique est peut-être l'exemple morphologique par excellence, surtout quand on songe à La théorie des couleurs de Goethe.

39. Ces expressions sont de Goethe et de Humboldt qui les utiliseront pour parler du rôle de l'historien.

40. Logique des sciences de la culture, traduit par Jean Carro avec la collaboration de Joël Gaubert, Paris, Éditions du Cerf, 2007, abrégé LSC ; Essai sur l'homme, Norbert Massa (trad.), Paris, Minuit, 1975 ; Le Mythe de l'État, Bertrand Vergely (trad.), Paris, Gallimard, 1993.

41. Ernst Cassirer, LSC, p. 202 : «Les doutes et les objections que l'on peut concevoir à l'endroit de la culture gardent toute leur importance. On doit reconnaître et concéder qu'elle n'est pas un tout qui se déploie harmonieusement, mais qu'elle est en proie aux plus grandes contradictions internes. La culture est "dialectique”, aussi vrai qu'elle est dramatique. Elle n'est pas un simple événement, un paisible déroulement, mais elle est une manière d'agir qui doit sans cesse se remettre à l'ouvrage et n'est jamais sûre de son but. Aussi ne peut-elle jamais se laisser aller tout bonnement à un optimisme naif ou à une croyance dogmatique en la "perfectibilité" de l'homme".

42. Patrice Maniglier, «L'humanisme interminable de Claude Lévi-Strauss », Les Temps Modernes, $n^{\circ}$ 609, juin-août 2000, p. 216-241.

43. Ernst Cassirer, La philosophie des formes symboliques, t. III, La phénoménologie de la connaissance, Claude Fronty (trad.), Paris, Minuit, 1972, p. 55 : «Devant l'échec de toute tentative visant à transcender purement et simplement le domaine de la forme, il ne faut pas se borner à toucher à ce domaine ici ou là, mais l'arpenter d'un bout à l'autre. Faute de pouvoir saisir directement l'infini, la pensée doit explorer tout le champ du fini ».

44. On peut mentionner les vidéos suivantes, sans être exhaustif : Clapping with Stones, 2005 ; What we saw upon Awekening, 2006 ; Brick Sellers of Kabul, 2006 ; In transit, 2008. Voir la monographie Lida Abdul, Torino, hopefulmonster editore, 2007.

45. Aby Warburg, " "La naissance de Vénus" et "Le printemps" de Sandro Botticelli », in Essais Florentins, Sibylle Müller (trad.), Paris, Klincksieck, 1990, p. 47 et suiv.

\section{RÉSUMÉS}

En deçà du linguistic turn, qui réduit abusivement le langage à ses seules dimensions représentative et discursive et court ainsi le risque de tomber dans l'écueil de l'« intellectualisme », et au-delà de l'iconic turn, qui, par réaction, déconstruit tellement le sujet créateur et acteur que le domaine de l'éthique s'en trouve dangereusement ébranlé, avec la menace de sombrer cette fois-ci dans l'écueil de «l'esthétisme », l'anthropologie de l'homme en 
mouvement proposée conjointement par Cassirer et Warburg ouvre la possibilité d'une troisième voie pour le discours sur l'art, replacé dans le cadre d'une théorie de la culture plus globale d'où une nouvelle conception du sujet peut émerger. Sur le modèle des enquêtes ethnologiques centrées davantage sur le rituel que sur le mythe écrit, tous deux perçoivent originairement l'image comme l'expression (Ausdruck) polarisée d'affects opposés, et cela avant même de l'envisager aussi comme la présentation (Darstellung) de valeurs, motifs ou idées que l'artiste comme le spectateur peuvent davantage mettre à distance d'eux que ne l'autorise le niveau expressif pur du rituel magique. Selon cette anthropologie, art et pensée mythique participent à un processus culturel complexe qui les déborde ; la technique, les sciences, l'histoire, le droit, etc. interférant constamment pour former des aires relationnelles de projection où s'entre-écrivent différents langages, la mise en évidence des points de basculement d'une aire à une autre permettant de fournir les éléments d'un diagnostic critique.

Cette «science générale de la culture comme théorie de l'homme en mouvement » se développe selon trois axes intrinsèquement liés qui organiseront notre propos. Elle est à un premier niveau une sémiotique des images, le schéma corporel fonctionnant comme médium symbolique originaire pour créer et recréer les images perçues. À un deuxième niveau, elle enracine continuellement le processus de création du sens des images dans une symbolisation rituelle. Enfin, elle implique à un dernier niveau une Kulturkritik ou, pour le dire autrement, comme c'est aussi le cas de manière emblématique chez Foucault, elle ouvre sur un "diagnostic critique » implicite qui met au jour le pouvoir de l'image en exhibant les points critiques où le principe d'un groupe de transformation d'images se modifie.

\section{INDEX}

Personnes citées : Cassirer (Ernst), Warburg (Aby), Goethe (Johann Wolfgang von)

Mots-clés : théorie de l'image, art et mouvement, iconologie, art et rituel, symbolisation rituelle, mémoire collective, mémoire iconique, anthropologie de l'art, culture, signification de l'image, fonction symbolique

\section{AUTEUR}

\section{MURIEL VAN VLIET}

Université Rennes 1, PhiCo-ExeCo, CEPA, université Paris 1, groupe de recherche « Les morphologies » (D. Cohn). Normalienne, agrégée de philosophie, docteur en philosophie, avec une thèse sur le thème de la forme selon E. Cassirer, elle a publié La forme selon Ernst Cassirer : de la morphologie au structuralisme, en 2013, aux Éditions des Presses universitaires de Rennes, dans la collection Æsthetica. 